\title{
Hello again, ANOVA: rethinking ANOVA in the context of confirmatory data analysis
}

Short title: rethinking analysis of variance

Haiyang Jin*

Department of Psychology, New York University Abu Dhabi

*Correspondence concerning this article should be addressed to Haiyang Jin, New York

University Abu Dhabi, Saadiyat Island, Abu Dhabi, United Arab Emirates. E-mail:

haiyang.jin@outlook.com

\section{Author Contributions}

H. Jin is the sole author of this article and is responsible for its content.

\section{Acknowledgement}

I am indebted to Yuzhu Ji for the conversations that inspired the motivation for this article. I especially would like to thank William G. Hayward for his expertise and feedback for the early version of this article. 


\section{Declaration of Conflicting Interests}

The author declared that there were no conflicts of interest with respect to the authorship or the publication of this article.

Open Practices Open Data: not applicable

Open Materials: https://osf.io/269rm/

Preregistration: not applicable

All materials have been made publicly available via OSF and can be accessed at https://osf.io/269rm/. 


\begin{abstract}
Analysis of variance (ANOVA) is one of the most popular statistical methods employed in psychological research. Despite its widespread usage, the appropriateness of the ways in which ANOVA is used in practice is not well-discussed. For instance, ANOVAs are frequently used as exploratory data analysis, even in confirmatory studies with explicit research questions and hypotheses. Such misapplication may invalidate some ANOVA conventions, resulting in reduced statistical power of detecting effects of interest, and even threatening the validity of research conclusions.
\end{abstract}

There are many widely-used ANOVA conventions, such as conducting post-hoc and simple effect analysis only when the main effect and interaction are significant, performing more ANOVAs by collapsing or separating one factor depending on the statistical significance of a higher-order interaction, and so on. This paper first evaluates the appropriateness of these conventions, then discusses the potential motivations possibly misunderstood by researchers, and provides practical suggestions. Moreover, this paper proposes to control the Type I error rate with Hypothesis-based Type I Error Rate (HER). Not only does HER consider the number of tests, but it also emphasizes the logical relationships among tests in rejecting the null hypothesis of research interest. Furthermore, this paper uses concreate examples to introduce the simple interaction analysis. For many studies, this technique can employ the most straightforward interaction to test effects of interest.

This paper additionally reviews ANOVA limitations and its alternative methods. Preregistration and open science are recommended to provide clarity for the selection of appropriate ANOVA tests in both confirmatory and exploratory studies. 
Key words: analysis of variance; ANVOA conventions; confirmatory data analysis; hypothesis-based Type I error rate; simple interaction analysis 


\section{Introduction}

When conducting an experiment, we are seldom interested in experiment-specific effects. Instead, we are more likely to be interested in effects that could be generalized to other similar participants and stimuli. Therefore, we need inferential statistics to infer population effects from observed results. One of the most popular inferential statistical methods, especially in psychological and other field studies (e.g., Weissgerber et al., 2018) with factorial designs, is analysis of variance (ANOVA). Almost all statistics textbooks and courses incorporate an introduction to ANOVA. ANOVA and its variants, e.g., repeatedmeasures ANOVA, are also widely implemented in commercial and open-source statistical software, such as SPSS, Jamovi, and JASP. With several clicks, researchers can obtain the results of their main effects and interactions, as well as post-hoc tests and simple effects analysis. Some of these results (mainly statistically significant ones) will be reported in publications to answer research questions. Despite the widespread usage of ANOVA, the previous literature rarely considers the appropriateness of the way in which ANOVA is applied to experimental data. Are all these conventional usages of ANOVA appropriate? If yes, what are the underlying motivations? If not, what are the appropriate alternative applications? One possible reason these questions persist is that statisticians mainly devote themselves to developing and optimizing statistical methods (e.g., ANOVA) and recommend typical routines for general purposes, but these recommendations may fail when it comes to specific research questions. Also, most researchers may put particular weight on conventions that are widely followed in the way that ANOVAs are implemented but overlook the appropriateness of those conventions.

This paper will not review the fundamental rationale and mathematical foundations underlying ANOVA, which have already been well articulated in many excellent books (e.g., 
Field, 2018; Rutherford, 2013; Wilcox, 2016). Instead, this paper will focus on discussing the appropriateness of several ANOVA conventions as used by researchers and will suggest alternative methods to address specific research questions. First, this paper revisits ANOVA in the context of exploratory and confirmatory data analysis. Second, this paper evaluates the appropriateness of some popular ANOVA conventions, discusses the underlying motivations potentially misunderstood by researchers, and provides alternative suggestions when needed. Third, some of the ANOVA limitations are reviewed, and alternative methods are briefly introduced. Finally, pre-registration and open-science are recommended to facilitate the use of appropriate ANOVA methods. Although the main focus of this paper is ANOVA, much of the following discussion also applies to its variants, e.g., repeated-measures ANOVA, and other statistical methods, e.g., linear mixed-effects models and Bayesian hierarchical models.

\section{Is ANOVA exploratory or confirmatory data}

\section{analysis?}

There are two main approaches in data analysis: exploratory and confirmatory. Exploratory data analysis (EDA) is "detective work" (Tukey, 1977) and empowers researchers with great flexibility to explore the potentially interesting relationships manifested by the data. By contrast, confirmatory data analysis (CDA) is more about confirming (or rejecting) hypotheses derived from theory (Rutherford, 2013). These two approaches work together to advance the sciences. On the one hand, CDA directly examines the theory-driven hypotheses and provides relatively more reliable answers to the questions of interest, facilitating theory revision and development. On the other hand, EDA helps to examine the unexplored sections that are missed by the theory-driven hypotheses and brings 
forth possible hypotheses for further studies. EDA is particularly critical as the first step of scientific investigation. Critically, we need both EDA and CDA (Tukey, 1969, 1980).

Is ANOVA an EDA or CDA? Maybe this is a wrong question in the first place. Nevertheless, the answer to this question is simple: ANOVA can be applied as either of them. The key feature to distinguish between these approaches is whether a specific research question is used to guide the analysis.

Considering that ANOVA can be used as either EDA or CDA, how are they applied practically in psychological experiments? The typical routine of performing ANOVA is to conduct the full factorial ANOVA at first (i.e., with all main effects and interactions among them). Then the post-hoc and simple effect analyses are performed when the corresponding main effects and interactions are significant, respectively. In addition, some researchers may also separate or collapse one or more factors depending on whether the higher-order interaction is significant or not, especially in ANOVA with more than two factors. Notably, there are no specific research questions or hypotheses guiding the analysis during this whole classical procedure. Therefore, ANOVA employed in the above application should be an EDA (rather than CDA).

The above classical application of ANOVA as EDA has been widely employed by researchers, even in confirmatory studies with specific questions and hypotheses. This misapplication can potentially impact the reliability and credibility of the evidence, and the extent of influence depends on whether the hypotheses are formulated before or after seeing the EDA results. If the hypotheses were proposed before conducting EDA, researchers might waste research resources on some effects that are not necessary for testing their hypothesis. Also, the misuse, in this case, may result in reduced statistical power for examining the effects of interest. By contrast, if the hypotheses were formulated after seeing EDA results 
(aka, Hypothesizing After the Results are Known; HARKing; Kerr, 1998), the influence of the misapplication is detrimental. This questionable research practice can translate Type I errors into theory, fail Popper's criteria of disconfirmability, and mistakenly take the values of prediction as those of accommodation (more see Kerr, 1998; Rubin, 2020). In any case, the classical convention of conducting EDA with specific research questions and hypotheses is not appropriate. Researchers should be clear about the approach (i.e., EDA or CDA) they intend to use for each question and employ the corresponding tests accordingly.

\section{Basic concepts in ANOVA}

In order to facilitate later discussion, I will use artificially-generated data to briefly recap some of the popular effects typically examined using ANOVA: main effects, interactions, post-hoc, and simple effects analysis. In this hypothetical study, let us assume that researchers proposed two protocols to train participants' face recognition abilities and randomly assigned participants into one of three groups: two training and one control group. Participants' performance of recognizing faces was measured before and after the training or the control task (more see Appendix A). Following the classical routine, a 3 (Group: control, Protocol 1, vs. Protocol 2) $\times 2$ (Test: pre-test vs. post-test) mixed ANOVA would be conducted. The primary outputs from this ANOVA that researchers would usually examine are the main effects and the interaction. Main effects test whether at least one level is different from others. The null hypothesis (i.e., $\mathrm{H}_{0}$ ) for testing the main effect is that all levels are comparable (e.g., $\mu_{1}=\mu_{2}=\mu_{3}$ ). For example, the main effect of Group tests whether there is evidence that average performance across pre- and post-tests of at least one group is different from the others. On the other hand, the interaction is only relevant when there is more than one factor involved in an ANOVA. In general, the interaction tests whether 
differences among levels of one factor are different for distinct levels of another factor(s), i.e., the difference of differences. For instance, the interaction between Group and Test could examine whether the differences between pre- and post-tests of at least one participant group are unequal from those of other groups.

In addition to the main effects and interaction, researchers usually also conduct simple effects and/or post-hoc analyses to perform follow-up comparisons. Specifically, simple effects analysis tests whether the levels of one factor are comparable at each level of the other variable. For instance, simple effects analysis of Group examines whether at least one group is different from others in the pre- and post-tests separately. These two simple effects analyses can be regarded as two one-way ANOVA with three levels (Rutherford, 2013). By contrast, post-hoc analysis tests pairwise comparisons. For example, post-hoc analysis for the main effect of Group could test differences in average face-recognizing performance across pre- and post-tests between the control and Protocol 1, control and Protocol 2, as well as those between Protocol 1 and Protocol 2. Since more than one comparison is examined in post-hoc and simple effect analysis, multiple comparison corrections are often employed to control the Familywise Type I Error Rate (FWER).

\section{Rethinking ANOVA conventions}

As discussed earlier, ANOVAs can be used as either EDA or CDA. Applying ANOVA for mismatched purposes may result in reduced statistical power or inflated FWER, among other possibilities. Therefore, researchers need to take care to employ the appropriate tests based on their research questions. This section will discuss the appropriateness (or inappropriateness) of some popular ANOVA tests in the context of EDA and CDA and the 
potential motivations for ANOVA conventions, and will also recommend some alternative approaches to resolve these issues.

\section{1. "Perform Post-hoc analysis only for the significant}

\section{main effect"}

One of the popular conventions with ANOVA is that researchers perform post-hoc analysis only if the main effect is significant. The appropriateness of this convention depends on the specific research question and whether researchers intend to perform EDA or CDA. Suppose that in the above hypothetical training study, Researcher 1A is specifically interested in whether participants differ in their face-recognizing performance across pre- and post-tests depending upon whether they were trained with Protocol 1 or 2 . Researcher 1B only wonders whether at least one group performance across pre- and post-tests is different from other groups, and he/she is not interested in which pair of groups differ from each other.

Researcher $1 \mathrm{C}$ would like to explore which groups differ from each other in the average performance of pre- and post-tests. As we can see, with the same experimental design, researchers may raise different research questions. By convention, a $3 \times 2$ ANOVA and corresponding post-hoc analysis would be conducted regardless of specific research questions. However, as discussed earlier, this application may not be appropriate in all cases. Instead, appropriate effects should be examined according to the specific questions of each researcher. If some of the pairwise comparisons are sufficient to provide potential answers to the research question, researchers may and should only contrast these pairs regardless of the main effect result. For instance, Researcher 1A should directly contrast the average performance of pre- and post-tests for participants in Protocol 1 group with that in Protocol 2 group. If exploring whether at least one level is different from others is the only effect of interest, e.g., Researcher 1B, examining the main effect is adequate, and no post-hoc analysis 
is needed. Notably, the conventional approach for examining the main effect with potentially additional post-hoc analysis is suboptimal for Researcher 1A and 1B's questions due to either reduced statistical power or redundant work.

By contrast, if the ultimate aim is to explore which of all the pairs differ, as with Researcher 1C's question, the appropriateness of performing post-hoc analysis only for the significant main effect depends on the post-hoc methods employed. For example, a significant main effect is a prerequisite for employing the least significance difference method for pairwise comparison, while Tukey-Kramer and Dunnett's T3 method can be used irrespective of the statistical significance of the main effect results (Hothorn, 2016; Wilcox, 2016). Actually, if Tukey-Kramer is used only when the main effect is significant, the FWER of pairwise comparisons is smaller than the conventional level of 0.05 (Bernhardson, 1975; Wilcox, 2016) where the statistical power will be reduced. Therefore, researchers should not always perform post-hoc analysis only when the main effect is significant. Instead, it should depend on their research questions and the post-hoc methods they use.

\section{2. "Performing simple effects analysis only when the}

\section{interaction is significant"}

Another convention in applying ANOVA is that researchers only conduct simple effects analysis when the interaction is significant. Like the first convention, the appropriateness of this convention is also subject to specific research questions. Suppose that in the same hypothetical training study, Researcher $2 \mathrm{~A}$ is interested in whether the facerecognizing performance is higher in the post- relative to pre-tests for the two training groups. Researcher 2B only wonders whether the performance changes between pre- and post-tests in at least one group differ from others. According to their research interests, 
Research 2A may and should examine the performance difference between the pre- and posttests for participants trained with the two protocols separately, i.e., two of the simple effects. By contrast, Research 2B should examine the interaction between Group and Test, and no simple effects analysis is needed. Of note, neither of them needs to apply the convention of conducting simple effects analysis only when the interaction is significant. Is there any situation under which this classical application is appropriate?

Indeed, it is not necessary to examine simple effects if the interaction is not significant in some cases (see below). It first needs to be clarified that the reason for this convention is not because the statistical significance of the interaction is informative for the significance of simple effects analysis results. As discussed earlier (see Section 3), the interaction and simple effects analysis examine different effects, where the interaction tests the difference of differences while simple effects test differences between particular conditions. More critically, a significant interaction does not guarantee that at least one of the simple effects would reach significance. Similarly, a non-significant interaction does not necessarily indicate that none of the simple effects would be significant. As shown with simulated results in Figure 1, the statistical significance of the interaction is not consistent with simple effects.

Then why or under which situations should we conduct simple effects analysis only when the interaction is significant? In some cases, the interaction and simple effects are both needed to provide evidence for directional effects or specific result patterns, as needed to test specific hypotheses in CDA. In other words, in these cases, we may draw conclusions only when the interaction and specific simple effects are both significant, and here it would be appropriate to inspect simple effects only when the interaction is significant. In the same vein, it is also appropriate to examine the interaction only when the simple effects are 
significant in these cases. Let us take a concrete example, the complete composite face task (Jin et al., under review; Richler \& Gauthier, 2014), to explain it further.

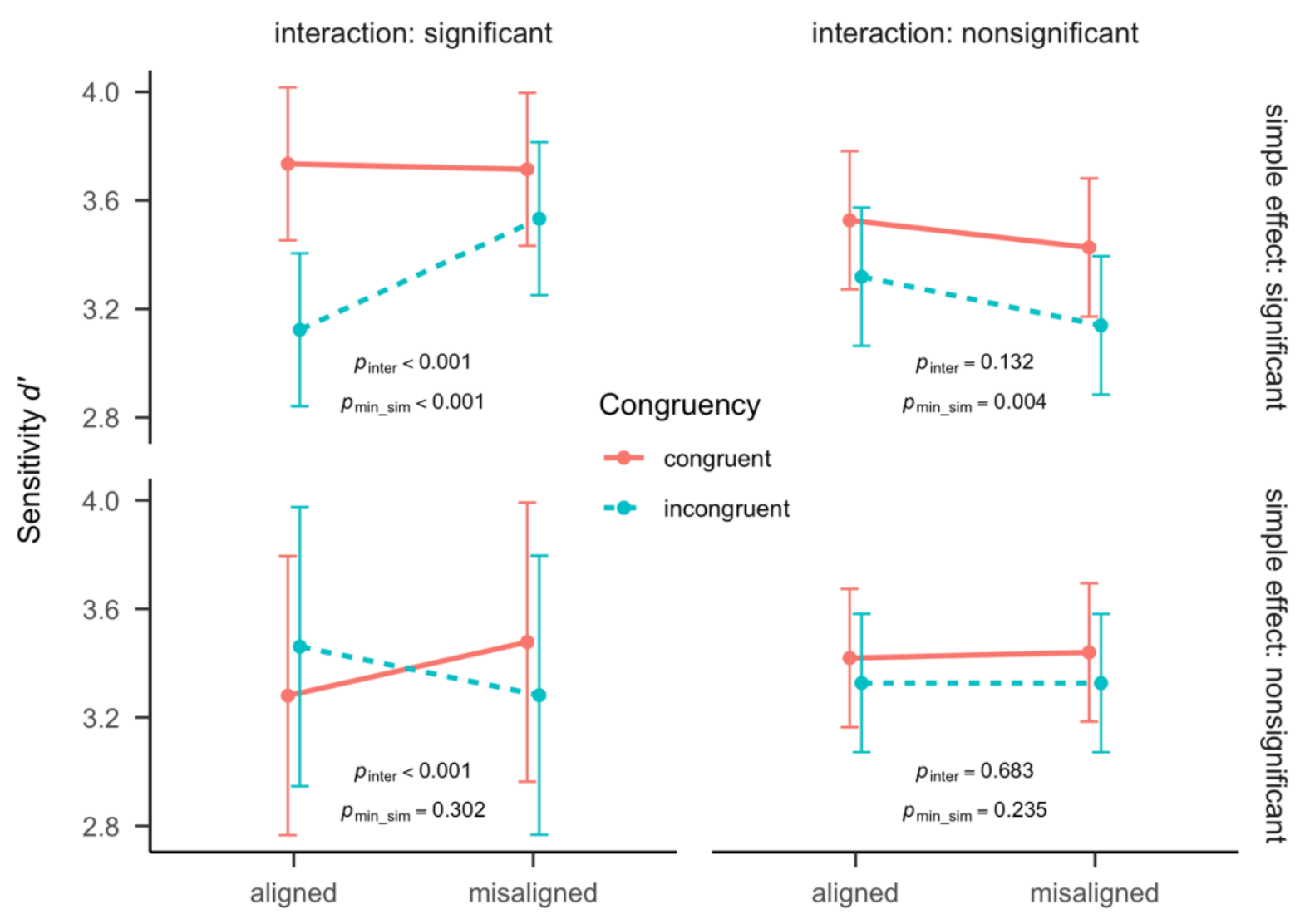

Alignment

Figure 1. Relationships between the statistical significance of interaction and simple effects with simulated data for the complete composite face task (Jin et al., under review; Richler \& Gauthier, 2014). The complete composite task employs a 2 (Congruency: congruent vs. incongruent) $\times 2$ (Alignment: aligned vs. misaligned) within-subjects design (see Appendix B for detailed descriptions). The left and right columns display results that the interaction is and not significant, respectively. The top row displays results that at least one of simple effects are significant. The bottom row displays results where none of simple effects is significant. " $p_{\text {inter" }}$ " denotes the p-value of the interaction and " $p_{\text {min_sim }}$ " denotes the smallest p-value among all simple effects. All p-values are not corrected. Error bars denote the $95 \%$ confidence intervals.

The complete composite face task is one of the popular paradigms measuring holistic face processing. In this task, two of the key independent variables are Congruency (congruent 
vs. incongruent) and Alignment (aligned vs. misaligned), and typically a $2 \times 2$ (repeatedmeasures) ANOVA is employed (see Appendix B for more details). The performance on congruent trials is expected to be better than on incongruent trials, which is called the Congruency effect (e.g., Richler et al., 2011). Moreover, this Congruency effect on aligned trials is also expected to be larger than that on misaligned trials (e.g., Richler et al., 2011). One typical result pattern is depicted in the top left panel of Figure 1, with which we may claim that the composite effect is observed. Which statistical tests should be conducted to examine the composite effect? The interaction between Congruency and Alignment is typically believed to be the index of the composite effect and has been widely employed in the literature (Jin et al., under review; Richler et al., 2011; Ross et al., 2015). Indeed, when the composite effect is observed, we should find this significant interaction. However, a significant interaction between Congruency and Alignment does not guarantee that we will observe the composite effect (e.g., the result pattern in the bottom left panel of Figure 1). This is partly because the significant interaction only indicates that the Congruency effect differs in aligned and misaligned conditions, but ANOVA obscures the direction of this difference. Also, the significant interaction does not inform us about the Congruency effect in the aligned condition. To obtain the necessary and sufficient conditions for claiming the composite effect, we should observe that (1) the Congruency effect is larger than zero in the aligned condition, i.e., one of the simple effects, and (2) the Congruency effect in the aligned condition is larger than that in the misaligned condition, i.e., the interaction with the particular direction. In other words, we should not claim to observe the composite effect if either the simple effect or the interaction fails to reach significance. Since we typically inspect the significance of the interaction at first, it is not necessary to continue to conduct simple effects analysis if the interaction is already not significant; we could not claim the composite effect regardless of the simple effects results. Although at least two tests are 
necessary for claiming the composite effect in the above example, researchers should not apply classical multiple comparison corrections. Since both tests have to be significant for claiming the composite effect, the Type I error rate for claiming the composite effect is already smaller than 0.05 when the conventional alpha of 0.05 is applied to the single tests (more see Section 4.4 and Appendix C).

The above composite face paradigm and composite effect are not unusual except that an explicit research hypothesis is proposed. Similar designs and expectations of directional effects can also be found in other confirmatory studies. For instance, in a training study with one control and training group who complete both the pre- and post-tests, for claiming that the training is effective, researchers should observe (1) better performance in the postrelative to pre-test in the training group (i.e., one of the simple effects) and (2) larger improvement in the training group than the control group (i.e., the interaction with the particular direction). Therefore, like the composite effect, both the simple effects and interaction should be tested in this training study. The training may be claimed to be effective only when both effects are statistically significant.

In summary, depending on the circumstances, researchers could test only the interaction, only the simple effects, or both of them, when using CDA with the guidance of their specific research questions. In EDA, researchers may test all these effects but they should be clear about whether the claimed effects are drawn from significant simple effects only or both significant simple effects and interaction results. Caution needs to be used if only simple effects are considered for testing a hypothesis when an interaction is involved in the experimental design. One should not claim that two effects differ from each other without directly comparing them, even when one is significantly different from 0 while the other is not (Gelman \& Stern, 2006; Makin \& Orban de Xivry, 2019; Nieuwenhuis et al., 2011; Palfi \& Dienes, 2020). For example, training should not be claimed effective only with the 
evidence that training and control groups differ in the post-, but not the pre-, tests. If researchers would like to draw conclusions that certain effects were observed in one, but not the other, condition, it is very likely that they also should test the differences between these effects in the two conditions, i.e., the interaction. Thus, in addition to the simple effects, the interaction between Group (training vs. control) and Test (pre- vs. post-tests) should also be tested to examine the effectiveness of training.

\section{3. "Collapsing or separating factors based on higher-}

\section{order interaction results"}

The other convention is to perform ANOVAs by collapsing or dividing one or more factor depending on the statistical significance of a higher-order interaction. This classical application could be regarded as an extension of the last one. Similar to relationships between a two-way interaction and simple effects, an interaction and its higher-order interaction also test different effects or hypotheses. Critically, results of a higher-order interaction are not informative for those of a lower-order interaction. Therefore, we should not collapse or divide factors and conduct more ANOVAs with this data-driven approach, i.e., depending on the statistical significance of a higher-order interaction. However, this does not mean researchers should not collapse and divide factors to conduct multiple ANOVAs. These decisions could be made based on specific research questions in confirmatory studies. For instance, Wang and colleagues (2019) employed the complete composite task to examine whether Asian observers showed composite effects for own-race, other-race, and otherspecies faces. Although the experimental design is 3 (Face category: own-race, other-race, vs. other-species $) \times 2$ (Congruency: congruent vs. incongruent $) \times 2$ (Alignment: aligned vs. misaligned), the interaction between Congruency and Alignment was tested for each face 
category separately to examine whether the composite effect was observed for those faces regardless of the statistical significance of the three-way interaction.

Moreover, similar to the composite face effect which needs to examine both a simple effect and interaction, we may also need to employ simple effects, two-way interaction, and three-way interaction together to conclude effects of interest in some confirmatory studies. Suppose we would like to test whether the composite effect for own-race faces is larger than that for other-race faces. Typically, a 2 (Race: own vs. other) $\times 2$ (Congruency: congruent vs. incongruent $) \times 2$ (Alignment: aligned vs. misaligned) within-subjects design would be employed. If the composite effect for own-race faces is larger than that for other-race faces, we should observe a significant three-way interaction. However, like testing the composite effect, a significant three-way interaction does not guarantee larger composite effects for own- relative to other-race faces. In addition to the three-way interaction, we also need to examine the composite effect for own-race faces, i.e., testing both the interaction between Congruency and Alignment and the Congruency effect in the aligned condition for own-race faces. In this example, to compare composite effects between different race faces, we need to test one three-way interaction, one two-way interaction, and one simple effect. We may claim larger composite effects for own- relative to other-race faces only when all these three effects are significant, and the difference directions are as expected. In other words, if the three-way interaction is not significant, it is not necessary to test the two-way interaction and simple effect anymore for claiming stronger composite effects for own-race faces. Likewise, it is unnecessary to test the three-way interaction if either the two-way interaction or the simple effect is not significant.

Suggestions for testing interactions and simple effects apply here as well. Researchers may conduct more ANOVAs by collapsing or separating factors according to research questions in confirmatory studies. However, these decisions should not be based on the 
statistical significance of a higher-order interaction. In addition, researchers also need to be clear about evidence used for claiming effects, especially in exploratory studies; for example, whether claimed effects are concluded from the three-way interaction only or the three-way interaction and other effects.

\subsection{Report "all" results}

It is also a convention that researchers (are asked to) report all results that could be tested by ANOVAs, including all main effects and interactions. Above, I have argued that specific effects should be tested according to the relevant research questions, especially in confirmatory studies. It is very unlikely that all effects are needed for testing hypotheses (except in EDA), especially in experiments with more than two factors. One may argue that reporting all results is useful because readers may be interested in effects other than those of interest to the experimenter. In this case, reporting all results in the supplementary materials would be more appropriate, as not all effects would be used to answer the research question in the original paper. Logically, if researchers are supposed to report all results, in addition to main effects and interactions, they should also report all post-hocs, simple effects, ANOVA results that have been separated for each factor, and even other effects that can be tested in ANOVA (see Section 4.6). However, such a report would quickly become unfeasible, particularly when more factors are employed in the experimental design. In the era of open science, a more efficient solution would be for researchers to share their data publicly rather than attempting to report all effects in the paper itself.

\subsection{Multiple comparison corrections}

Probably the most popular justification for performing ANOVA instead of multiple ttests in comparing more than two means is that ANOVA can control the Type I error rate to 
an acceptable level (e.g., 0.05), whereas multiple t-tests inflate this error rate. This error rate is known as FWER, referring to the probability that at least one of the comparisons is significant when null hypotheses for all these tests are true (Frane, 2021; Tukey, 1953). By contrast, another Type I error rate is the Single Test Type I Error Rate (STER), which refers to the probability of a single test being significant when the null hypothesis is true. It is noteworthy that although multiple t-tests inflate FWER, STER for each comparison still remains at the acceptable level.

Should we control the Type I error rate at the single test or familywise level? Again, an answer depends on the specific research question being asked. Specifically, it depends on the number of tests needed for hypothesis testing and the logical relationships among these tests in rejecting the null hypothesis of research interest. When only one comparison is needed to test the hypothesis, we may control the Type I error rate with STER, which also matches FWER in this case. When more than one comparison is needed to test the hypothesis, and the null hypothesis would be rejected as long as at least one of them is significant, we should control the Type I error rate with FWER, e.g., Bonferroni, Tukey, or Dunnett's methods (Frane, 2021; Wilcox, 2016). When more than one comparison is needed to test the hypothesis and the null hypothesis would be rejected only when all tests are significant, controlling the Type I error rate with FWER or STER would be too stringent. Since multiple tests need to be significant in order to reject the null hypothesis of research interest, this Type I error rate could be defined as Conjunction Type I Error Rate (CER), which refers to the probability that all tests are significant when null hypotheses for all these tests are true. For instance, as discussed in Section 4.2, both the interaction and one simple effect should be examined for claiming the composite face effect. Applying STER of 0.05 for the two single tests leads to a CER below 0.025 (see Appendix C). For this case, we may apply STER of 0.1 to control the Type I error rate for claiming the composite effect, i.e., 
CER, below 0.05 (see Appendix C). Type I error rates applied in the above three scenarios could be summarized as the Hypothesis-based Type I Error Rate (HER), which refers to the probability of rejecting the null hypothesis of research interests (and claiming certain effects) when the null hypotheses of all single tests are true.

In addition to STER, FWER, CER, and HER, a higher level Type I error rate is the Study-wise Type I Error Rate (SWER, or Experiment-wise Type I error rate; Frane, 2021; Tukey, 1953). SWER is the probability of rejecting at least one of the null hypotheses for research interests when all these null hypotheses are true in one study. SWER matches HER if only one hypothesis of research interest is tested in the study. If more than one hypothesis is examined in a single study, researchers may apply multiple comparison corrections, e.g., Bonferroni corrections, to control the Type I error rate at the study level.

These different Type I error rates naturally bring the question: which of them should we control (to an acceptable level) in our research? It should be noted that controlling Type I error rate to different levels does not alter the statistical results (e.g., F-values and effect sizes). It is the criteria in dichotomizing results as statistically significant versus nonsignificant that changes, i.e., the alpha. Therefore, instead of asking at which level the Type I error rate should be controlled, we should probably ask ourselves at which level we would like to control the Type I error rate. In other words, we should justify our alpha (Lakens et al., 2018). Some researchers may feel that choosing alpha in individual studies is an arbitrary decision. However, we first have to admit that the conventional alpha of 0.05 is arbitrary (e.g., McElreath, 2020). Also, any researcher has to implicitly choose the alpha level as influenced by multiple comparisons. When multiple comparison corrections are employed, we implicitly choose to control the FWER to an acceptable level, during which we control STER more stringently. Furthermore, even if researchers feel uncomfortable in defining (or justifying) the alpha in their studies and decide to control STER for all tests, they are still 
choosing alpha. In summary, we should make our decisions regarding alpha more explicitly and can do so by defining and justifying alpha based on our specific research questions.

Researchers should control the Type I error rate with HER in confirmatory studies. Specifically, first, researchers should pre-define which tests need to be conducted to test the hypothesis. For instance, both the interaction between Congruency and Alignment and the Congruency effect in the aligned condition (i.e., one simple effect) should be tested for examining the composite effect. Second, researchers should clarify the logical relationships among the tests in rejecting the null hypothesis of research interests: the composite effect could be claimed only when both the interaction and the simple effect are significant. Third, researchers should set HER for rejecting the null hypothesis of research interest and corresponding STER for each test. For example, HER for claiming the composite effect could be set at 0.05 and, therefore, STER for the interaction and the simple effect would be set as 0.1 (see Appendix C).

For EDA, a similar procedure applies as well. The question in EDA could be described as whether there is any effect examined by ANOVAs reaching statistical significance. First, all effects need to be examined in EDA if no plans are pre-defined. For example, in a $2 \times 3$ factorial design, EDA needs to test two main effects and one interaction if the post-hoc, simple effect and other analyses (see Section 4.6) are not included in this EDA. Second, the corresponding null hypothesis of research interest is that all three tests are not statistically significant. This hypothesis would be rejected as long as one of tests reaches significance. Third, when the conventional alpha of 0.05 is set as the HER, the corresponding STER for each of the three tests would be about 0.017 . By contrast, if the STER is set as 0.05, HER (or FWER) for observing at least one significant effect could be as high as 0.14 (Cramer et al., 2016), even when this EDA does not include post-hoc, simple effect, and other analyses. 
Experimenters should define and justify their own alpha. Readers should pay attention to at which level conclusions are drawn in the paper and even may apply different alpha when necessary. It is more important to keep in mind that statistical significance is not everything (Amrhein et al., 2019; Ioannidis, 2019; Lykken, 1968; Sterne, 2001; Wasserstein et al., 2019).

\subsection{Simple interaction analysis and beyond}

In the above hypothetical training study, probably the most reasonable research questions are (1) whether Protocol 1 is effective, (2) whether Protocol 2 is effective, and (3) which protocol is more effective. Since a control group is included in this study to exclude the general performance increases that are not credited to training, to answer the first two questions, one of the effects researchers need to test is whether performance increases from pre- to post-tests is higher for the training group than the control group, i.e., the difference of differences. Unfortunately, this difference of differences is not tested by any effect researchers typically obtained from a $3 \times 2$ ANOVA; that is, the main effects, the $3 \times 2$ interaction, post-hoc tests, or simple effect analysis results. As discussed earlier, this effect of interest should be tested by a $2 \times 2$ interaction. For instance, the 2 (Protocol 1 vs. control) $\times 2$ (pre-test vs. post-test) interaction should be used to examine the effectiveness of Protocol 1. Like testing the composite face effect, a significant $2 \times 2$ interaction does not necessarily indicate that Protocol 1 is effective. Researchers also should examine whether the performance is better in the post- than pre-tests for participants trained with Protocol 1, i.e., one of the simple effects (researchers may also set the CER to control the appropriate Type I error rate). In the same vein, the other two questions should be examined by the 2 (Protocol 2 vs. control) $\times 2$ (pre-test vs. post-test) interaction and the $2($ Protocol 1 vs. Protocol 2$) \times 2$ 
(pre-test vs. post-test) interaction, as well as the corresponding simple effects, respectively (see Appendix A for an analysis example).

The $2 \times 2$ interaction in a $3 \times 2$, or more complicated, experimental design can be employed to test some effects of interest. Examining this kind of $2 \times 2$ interaction could be termed as simple interaction analysis, which is the simplest and most straightforward interaction for hypothesis testing. In a $2 \times 2$ experimental design, the simple interaction matches the highest-order interaction. In a $2 \times 2 \times 2$ experimental design, simple interaction analysis could examine six effects by separating each of the three factors. Of particular note, the statistical significance of the interaction with all conditions is not a prerequisite for performing simple interaction analysis. Again, whether simple interaction analysis should be performed or how it should be performed (e.g., which factor should be separated) solely depends on the research questions.

Simple interaction analysis, in addition to main effects, other interaction, post-hoc tests and simple effect analysis, does not cover all the effects researchers may be interested in in a study. For instance, in the hypothetical training study, researchers may wonder whether the average performance increases of the two protocol groups are higher than the control group. None of the effects mentioned above could be employed to answer this question. In this case, the experimenter should apply custom contrasts (specifically, a custom contrast of $c(1,-1,-.5, .5,-.5, .5)$ should be applied to the control pre-test, control post-test, Protocol 1 pre-test, Protocol 1 post-test, Protocol 2 pre-test, and Protocol 2 post-test conditions, respectively; see Appendix A for an analysis example). Similarly, researchers may have other research questions that cannot be examined with such frequently used effects. We should not ignore or underestimate the importance of custom contrasts in statistical analyses. 


\section{Limitations of ANOVAs}

Like all other statistical methods, ANOVA has its limitations. A good understanding of these limitations enables a researcher to be aware of when and the extent to which any of them may jeopardize the validity of conclusions that are drawn from their results. First, ANOVA is only able to incorporate one random effect, which is usually participants. However, apart from the random effect of participants, other random effects, such as stimuli (or items) and schools, also should be considered in psychological experiments (Judd et al., 2012). When random effects solely include participants, ANOVA results could only be generalized to other similar participants, but not to, for instance, other similar stimuli. Second, ANOVA can only analyze data whose independent variables are categorical. When a continuous variable is intended to be incorporated as an independent variable in ANOVA, it is usually converted into a discrete variable with some custom criteria, which may lose valuable information. Moreover, the dependent variable in ANOVA should be an unbounded continuous variable. When ANOVA is employed to analyze accuracy, bounded by 0 and 1 , or ordered discrete responses, the results may be invalidated (Liddell \& Kruschke, 2018). Furthermore, participants who have missing values in at least one condition have to be discarded if ANOVA is used. Discarding data due to missing values is not only a waste of experimental resources, which potentially reduces statistical power, but may also lead to biased results without considering information from all samples (Brauer \& Curtin, 2018; Kliegl et al., 2011; Linck \& Cunnings, 2015; Matuschek et al., 2017). 


\section{Alternatives of ANOVAs}

Indeed, ANOVA is a relatively straightforward and efficient statistical method. Nevertheless, researchers should be cautious about the above limitations and the underlying assumptions. When the validity of ANOVA results is potentially threatened by its limitations or assumption violations, researchers need to employ alternative methods. Frequentist and Bayesian hierarchical models (Kruschke \& Liddell, 2018), also known as mixed-effects models or multilevel models, are the most popular alternatives to (repeated-measures) ANOVA. Not only are hierarchical models able to deal with the ANOVA limitations discussed earlier (Boisgontier \& Cheval, 2016; Brauer \& Curtin, 2018; Bürkner \& Vuorre, 2019; Enders \& Tofighi, 2007; Finch et al., 2019; Jaeger, 2008, 2008; Judd et al., 2012, 2017; Kliegl et al., 2011; Liddell \& Kruschke, 2018; Matuschek et al., 2017), but they also release the homogeneity of variances and sphericity assumptions in (repeated-measures) ANOVA (Linck \& Cunnings, 2015; Magezi, 2015; West et al., 2015). Furthermore, hierarchical models provide more accurate estimations of population effects by considering the individual results in the context of others, i.e., shrinkage. There are numerous excellent books available to provide more details on this topic (e.g., Finch et al., 2019; Gelman et al., 2013; Kruschke, 2014; Lambert, 2018; McElreath, 2020; Stroup, 2012).

Most of the above discussion and suggestions for ANOVA also apply to Frequentist and Bayesian hierarchical models. No matter which statistical methods are used, researchers always need to be clear about their research questions and which effects should be examined to test the hypothesis. One of the advantages of using hierarchical models is that researchers may employ priori contrasts (Schad et al., 2020) to test the effects of interest and avoid follow-up comparisons. Another advantage is that researchers may use hierarchical models to perform one-sided tests to increase statistical power. Some readers may notice that strictly 
speaking, a significant interaction between Congruency and Alignment and the significant Congruency effects for aligned faces are not really the necessary and sufficient condition for claiming the composite effect. As described in the prediction, both the expected interaction and simple effects are directional; however, ANOVA obscures this directional information. Researchers may examine the descriptive statistical results to check the difference directions when ANOVA is employed. By contrast, in hierarchical models, researchers may perform one-sided tests to examine directional effects directly. Moreover, simple interaction analysis can also be applied in hierarchical models. For instance, simple interaction analysis in a Frequentist hierarchical model was employed in an electroencephalogram study to explore the dependency of the N170 face-specific components (i.e., the difference of N170 amplitudes between faces and non-face objects) on other factors (Jin et al., in preparation).

One caveat is that hierarchical models may not be friendly to EDA if researchers aim to explore all effects discussed earlier. Usually, more than one hierarchical model needs to be employed to test these effects without following-up comparisons. Each model may take some time depending on the model complexities and the amount of data. Sometimes researchers even need to deal with the failure to converge issue, which is not uncommon, especially in hierarchical models with the maximum random-effects structure (Barr et al., 2013; Matuschek et al., 2017).

\section{Pre-registration and registered reports}

The cornerstone of the above discussion is that researchers must be clear about the research questions and whether they plan to perform EDA or CDA. Considering the great flexibility in research practices (e.g., John et al., 2012; Simmons et al., 2011; Wicherts et al., 2016), pre-registration (Jonas \& Cesario, 2016), as an objective and persuasive approach to 
distinguish CDA from EDA (Chambers, 2019), should be employed. For CDA, researchers need to pre-register their research questions, statistical methods, effects that should be examined for each hypothesis, and logical relationships among these effects in rejecting the null hypothesis of research interest. Also, researchers need to pre-define (and justify) their alpha and report the corresponding HER and STER.

For EDA, researchers also need to pre-register similar information. Researchers can state explicitly in the pre-registration process if no research questions are proposed before data analysis. Also, researchers need to list all effects to be explored. As discussed earlier, the statistical significance should not be used as justifications for conducting more analysis. Therefore, researchers need to exhaust all effects they intend to examine, e.g., main effects, interaction, post-hocs, simple effects, simple interaction effects, and even some effects with custom contrasts, if no plan is made. Moreover, researchers also need to pre-define (and justify) the alpha. It is potentially reasonable to apply the alpha of 0.05 to single tests to increase the statistical power in EDA. However, it is also researchers' obligation to report the corresponding HER (or FWER) and remind readers of the tentative properties of these results before conducting confirmatory studies.

Researchers even may choose to conduct their study as a registered report (Nosek \& Lakens, 2014) or peer community in registered reports ${ }^{1}$ to make sure their study design is appropriate before data collection. Pre-registration of the above information, or pre-registered reports, will increase the transparency of study results. The field could consider incorporating

\footnotetext{
${ }^{1} \mathrm{https}: / /$ peercommunityin.org/
} 
pre-registration as one of the standard procedures in the scientific investigation, as has been advocated by supporters of Open Science.

\section{Summary}

This paper reviews the appropriateness of ANOVA tests in the context of CDA and EDA and clarifies the potentially misunderstood motivations for some ANOVA conventions. In general, it is not appropriate to make analysis decisions depending on the statistical significance of another effect, even in exploratory studies. Instead, these decisions should be based on specific research questions and analysis plans. Also, HER is proposed to control the Type I error rate. HER considers both the number of tests and their logical relationships in rejecting the null hypothesis of research interest. It directly describes the Type I error rate of claiming the effect of interest when the null hypotheses of all tests are true. For instance, when the null hypothesis of research interest can be rejected only if multiple tests are significant, as demonstrated in the composite face task, the Type I error rate should be controlled with CER. This paper employs concrete examples to introduce the simple interaction analysis, which can use the most straightforward interaction to test effects of interest. Researchers should be cautious about ANOVA assumptions and limitations and may employ alternative methods when necessary. Finally, pre-registration is recommended to preregister research questions, statistical methods, effects of interests, HER, STER, and other necessary information to provide clarity for the selection of appropriate ANOVA tests in both confirmatory and exploratory studies. 


\section{References}

Amrhein, V., Greenland, S., \& McShane, B. (2019). Scientists rise up against statistical significance. Nature, 567(7748), 305-307. https://doi.org/10.1038/d41586-019$00857-9$

Barr, D. J., Levy, R., Scheepers, C., \& Tily, H. J. (2013). Random effects structure for confirmatory hypothesis testing: Keep it maximal. Journal of Memory and Language, 68(3), 255-278. https://doi.org/10.1016/j.jml.2012.11.001

Bernhardson, C. S. (1975). Type I Error Rates When Multiple Comparison Procedures Follow a Significant F Test of ANOVA. Biometrics, 31(1), 229-232. https://doi.org/10.2307/2529724

Boisgontier, M. P., \& Cheval, B. (2016). The anova to mixed model transition. Neuroscience \& Biobehavioral Reviews, 68, 1004-1005. https://doi.org/10.1016/j.neubiorev.2016.05.034

Brauer, M., \& Curtin, J. J. (2018). Linear mixed-effects models and the analysis of nonindependent data: A unified framework to analyze categorical and continuous independent variables that vary within-subjects and/or within-items. Psychological Methods, 23(3), 389-411. https://doi.org/10.1037/met0000159

Bürkner, P.-C., \& Vuorre, M. (2019). Ordinal Regression Models in Psychology: A Tutorial. Advances in Methods and Practices in Psychological Science, 2(1), 77-101. https://doi.org/10.1177/2515245918823199

Chambers, C. D. (2019). The Seven Deadly Sins of Psychology. Princeton University Press. https://doi.org/10.2307/j.ctvc7742b

Cramer, A. O. J., van Ravenzwaaij, D., Matzke, D., Steingroever, H., Wetzels, R., Grasman, R. P. P. P., Waldorp, L. J., \& Wagenmakers, E.-J. (2016). Hidden multiplicity in 
exploratory multiway ANOVA: Prevalence and remedies. Psychonomic Bulletin \& Review, 23(2), 640-647. https://doi.org/10.3758/s13423-015-0913-5

Enders, C. K., \& Tofighi, D. (2007). Centering predictor variables in cross-sectional multilevel models: A new look at an old issue. Psychological Methods, 12(2), 121138. https://doi.org/10.1037/1082-989X.12.2.121

Field, A. (2017). Discovering statistics using IBM SPSS statistics (5th edition). SAGE Publications.

Finch, W. H., Bolin, J. E., \& Kelley, K. (2019). Multilevel Modeling Using R (2nd ed.). Chapman and Hall/CRC. https://doi.org/10.1201/9781351062268

Frane, A. V. (2021). Experiment-Wise Type I Error Control: A Focus on $2 \times 2$ Designs. Advances in Methods and Practices in Psychological Science. https://doi.org/10.1177/2515245920985137

Gelman, A., Carlin, J. B., Stern, H. S., Dunson, D. B., Vehtari, A., \& Rubin, D. B. (2013). Bayesian Data Analysis (3rd ed.). Chapman and Hall/CRC. https://doi.org/10.1201/b16018

Gelman, A., \& Stern, H. (2006). The Difference Between "Significant" and "Not Significant" is not Itself Statistically Significant. The American Statistician, 60(4), 328-331. https://doi.org/10.1198/000313006X152649

Hothorn, L. A. (2016). The two-step approach-A significant ANOVA F-test before Dunnett's comparisons against a control-Is not recommended. Communications in Statistics - Theory and Methods, 45(11), 3332-3343. https://doi.org/10.1080/03610926.2014.902225

Ioannidis, J. P. A. (2019). Retiring significance: A free pass to bias. Nature, 567, 461. 
Jaeger, T. F. (2008). Categorical data analysis: Away from ANOVAs (transformation or not) and towards logit mixed models. Journal of Memory and Language, 59(4), 434-446. https://doi.org/10.1016/j.jml.2007.11.007

Jin, H., Hayward, W. G., \& Corballis., P. (in preparation). A full N170 is generated once a face is perceived with high subjective confidence.

Jin, H., Oxner, M., Corballis, P., \& Hayward, W. G. (under review). Holistic face processing is influenced by non-conscious visual information.

John, L. K., Loewenstein, G., \& Prelec, D. (2012). Measuring the Prevalence of Questionable Research Practices With Incentives for Truth Telling. Psychological Science, 23(5), 524-532. https://doi.org/10.1177/0956797611430953

Jonas, K. J., \& Cesario, J. (2016). How can preregistration contribute to research in our field? Comprehensive Results in Social Psychology, 1(1-3), 1-7.

https://doi.org/10.1080/23743603.2015.1070611

Judd, C. M., Westfall, J., \& Kenny, D. A. (2012). Treating stimuli as a random factor in social psychology: A new and comprehensive solution to a pervasive but largely ignored problem. Journal of Personality and Social Psychology, 103(1), 54-69. https://doi.org/10.1037/a0028347

Judd, C. M., Westfall, J., \& Kenny, D. A. (2017). Experiments with More Than One Random Factor: Designs, Analytic Models, and Statistical Power. Annual Review of Psychology, 68(1), 601-625. https://doi.org/10.1146/annurev-psych-122414-033702

Kerr, N. L. (1998). HARKing: Hypothesizing after the results are known. Personality and Social Psychology Review, 2(3), 196-217. https://doi.org/10.1207/s15327957pspr0203_4

Kliegl, R., Wei, P., Dambacher, M., Yan, M., \& Zhou, X. (2011). Experimental effects and individual differences in linear mixed models: Estimating the relationship between 
spatial, object, and attraction effects in visual attention. Frontiers in Psychology, 1 , 238. https://doi.org/10.3389/fpsyg.2010.00238

Kruschke, J. K. (2014). Doing bayesian data analysis: A tutorial with R, JAGS, and Stan. https://doi.org/10.1016/b978-0-12-405888-0.09999-2

Kruschke, J. K., \& Liddell, T. M. (2018). The Bayesian New Statistics: Hypothesis testing, estimation, meta-analysis, and power analysis from a Bayesian perspective. Psychonomic Bulletin \& Review, 25(1), 178-206. https://doi.org/10.3758/s13423-016$1221-4$

Lakens, D., Adolfi, F., Albers, C. J., Anvari, F., Apps, M. A. J., Argamon, S. E., Baguley, T., Becker, R. B., Benning, S. D., Bradford, D. E., Buchanan, E. M., Caldwell, A. R., Van Calster, B., Carlsson, R., Chen, S.-C., Chung, B., Colling, L. J., Collins, G. S., Crook, Z., ... Zwaan, R. A. (2018). Justify your alpha. Nature Human Behaviour, 2(3), 168-171. https://doi.org/10.1038/s41562-018-0311-x

Lambert, B. (2018). A student's guide to Bayesian statistics. SAGE.

Liddell, T. M., \& Kruschke, J. K. (2018). Analyzing ordinal data with metric models: What could possibly go wrong? Journal of Experimental Social Psychology, 79, 328-348. https://doi.org/10.1016/j.jesp.2018.08.009

Linck, J. A., \& Cunnings, I. (2015). The Utility and Application of Mixed-Effects Models in Second Language Research. Language Learning, 65(S1), 185-207. https://doi.org/10.1111/lang.12117

Lykken, D. T. (1968). Statistical significance in psychological research. Psychological Bulletin, 70(3, Pt.1), 151-159. https://doi.org/10.1037/h0026141

Magezi, D. A. (2015). Linear mixed-effects models for within-participant psychology experiments: An introductory tutorial and free, graphical user interface (LMMgui). Frontiers in Psychology, 6, 2. https://doi.org/10.3389/fpsyg.2015.00002 
Makin, T. R., \& Orban de Xivry, J.-J. (2019). Ten common statistical mistakes to watch out for when writing or reviewing a manuscript. ELife, 8, 1-13. https://doi.org/10.7554/eLife.48175

Matuschek, H., Kliegl, R., Vasishth, S., Baayen, R. H., \& Bates, D. (2017). Balancing Type I error and power in linear mixed models. Journal of Memory and Language, 94, 305315. https://doi.org/10.1016/j.jml.2017.01.001

McElreath, R. (2020). Statistical Rethinking (2nd ed.). Chapman and Hall/CRC. https://doi.org/10.1201/9780429029608

Nieuwenhuis, S., Forstmann, B. U., \& Wagenmakers, E.-J. (2011). Erroneous analyses of interactions in neuroscience: A problem of significance. Nature Neuroscience, 14(9), 1105-1107. https://doi.org/10.1038/nn.2886

Nosek, B. A., \& Lakens, D. (2014). Registered reports: A method to increase the credibility of published results. Social Psychology, 45(3), 137-141. https://doi.org/10.1027/1864-9335/a000192

Palfi, B., \& Dienes, Z. (2020). Why Bayesian "Evidence for H1" in One Condition and Bayesian "Evidence for H0" in Another Condition Does Not Mean Good-Enough Bayesian Evidence for a Difference Between the Conditions. Advances in Methods and Practices in Psychological Science, 3(3), 300-308. https://doi.org/10.1177/2515245920913019

Richler, J. J., Cheung, O. S., \& Gauthier, I. (2011). Holistic processing predicts face recognition. Psychological Science, 22(4), 464-471. https://doi.org/10.1177/0956797611401753

Richler, J. J., \& Gauthier, I. (2014). A meta-analysis and review of holistic face processing. Psychological Bulletin, 140(5), 1281-1302. https://doi.org/10.1037/a0037004 
Ross, D. A., Richler, J. J., \& Gauthier, I. (2015). Reliability of composite-task measurements of holistic face processing. Behavior Research Methods, 47(3), 736-743. https://doi.org/10.3758/s13428-014-0497-4

Rubin, M. (2020). The Costs of HARKing. The British Journal for the Philosophy of Science. https://doi.org/10.1093/bjps/axz050

Rutherford, A. (2013). Anova and Ancova: A GLM Approach. John Wiley \& Sons, Inc. https://doi.org/10.1002/9781118491683

Schad, D. J., Vasishth, S., Hohenstein, S., \& Kliegl, R. (2020). How to capitalize on a priori contrasts in linear (mixed) models: A tutorial. Journal of Memory and Language, 110, 104038. https://doi.org/10.1016/j.jml.2019.104038

Simmons, J. P., Nelson, L. D., \& Simonsohn, U. (2011). False-positive psychology: Undisclosed flexibility in data collection and analysis allows presenting anything as significant. Psychological Science, 22(11), 1359-1366. https://doi.org/10.1177/0956797611417632

Sterne, J. A. C. (2001). Sifting the evidence-What's wrong with significance tests? Another comment on the role of statistical methods. BMJ, 322(7280), 226-231. https://doi.org/10.1136/bmj.322.7280.226

Stroup, W. W. (2012). Generalized Linear Mixed Models. CRC Press. https://doi.org/10.1201/b13151

Tukey, J. W. (1953). The problem of multiple comparisons. In H. I. Braun (Ed.), The collected works of John W. Tukey, Vol. VIII. Multiple comparisons: 1948-1983. Chapman \& Hall.

Tukey, J. W. (1969). Analyzing data: Sanctification or detective work? American Psychologist, 24(2), 83-91. https://doi.org/10.1037/h0027108

Tukey, J. W. (1977). Exploratory data analysis. Reading, MA: Addison-Wesley. 
Tukey, J. W. (1980). We Need Both Exploratory and Confirmatory. The American Statistician, 34(1), 23-25. https://doi.org/10.1080/00031305.1980.10482706

Wang, Z., Quinn, P. C., Jin, H., Sun, Y.-H. P., Tanaka, J. W., Pascalis, O., \& Lee, K. (2019). A regional composite-face effect for species-specific recognition: Upper and lower halves play different roles in holistic processing of monkey faces. Vision Research, 157, 89-96. https://doi.org/10.1016/j.visres.2018.03.004

Wasserstein, R. L., Schirm, A. L., \& Lazar, N. A. (2019). Moving to a World Beyond “ $p<$ 0.05.” The American Statistician, 73(sup1), 1-19. https://doi.org/10.1080/00031305.2019.1583913

Weissgerber, T. L., Garcia-Valencia, O., Garovic, V. D., Milic, N. M., \& Winham, S. J. (2018). Why we need to report more than "Data were Analyzed by t-tests or ANOVA.”ELife, 7, 1-16. https://doi.org/10.7554/eLife.36163

West, B. T., Welch, K. B., \& Galecki, A. T. (2015). Linear Mixed Models: A Practical Guide Using Statistical Software (2nd ed.).

Wicherts, J. M., Veldkamp, C. L. S., Augusteijn, H. E. M., Bakker, M., van Aert, R. C. M., \& van Assen, M. A. L. M. (2016). Degrees of Freedom in Planning, Running, Analyzing, and Reporting Psychological Studies: A Checklist to Avoid p-Hacking. Frontiers in Psychology, 7, 1832. https://doi.org/10.3389/fpsyg.2016.01832

Wilcox, R. R. (2016). Understanding and applying basic statistical methods using R. John Wiley \& Sons. 


\section{Appendix A}

\section{A hypothetical training study}

In this hypothetical training study, let us assume that two protocols were proposed to train participants' face recognition abilities. We would like to know (1) whether the first protocol is effective, (2) whether the second protocol is effective, and (3) which protocol is more effective. Two groups of participants were trained with Protocol 1 and 2, respectively, and a third group completed a control task. All participants' performance in recognizing faces was measured before and after the training (or the control task). In summary, it is a 3 (Group: control, Protocol 1, vs. Protocol 2) $\times 2$ (Test: pre-test vs. post-test) mixed experimental design.

Before performing any analysis, we need to clarify which effects should be examined for answering each question and the logical relationships among them in rejecting the null hypothesis of research interest:

1. For the first two questions, we need to examine (1) whether the post-test performance is better than the pre-test in the training group (e.g., Protocol 1), and (2) whether the performance increases (i.e., post-test - pre-test) in the training group (e.g., Protocol 1) are larger than the control group. We may claim that the training protocol (e.g., Protocol 1) is effective only when both tests are significant with particular difference directions.

2. For the third question, we need to examine (1) whether the performance increases in Protocol 1 group are different from Protocol 2 group, (2) whether the post-test performance is better than the pre-test in Protocol 1 group, and (3) whether the post-test performance is better than the pre-test in Protocol 2 group. We may claim that Protocol 1 is more effective if only (1) and (2) are significant with particular difference directions. We may claim that Protocol 2 is more effective if only (1) and (3) are significant with particular difference directions. If all three tests are 
Table A1

The ground truth for each condition.

\begin{tabular}{llll}
\hline Test & control & Protocol 1 & Protocol 2 \\
\hline pre-test & 3.00 & 3.00 & 3.00 \\
post-test & 3.50 & 3.70 & 4.30 \\
\hline
\end{tabular}

Note. Population parameters used to simulate

data for the hypothetical training study.

significant, we need to check the direction of the interaction to conclude which protocol is more effective.

The alpha level of 0.05 would be used at the single test level for all these effects, and, therefore, the Hypothesis-based Type I Error Rate (HER) is not higher than 0.05 (see Appendix C).

\section{Simulating data}

Let us simulate a data set for the above design with 30 participants for each group. Please refer to the codes in the open materials for detailed simulation procedures. Table A1 displays the ground truth for each condition, and the structure of the simulated data is as follows:

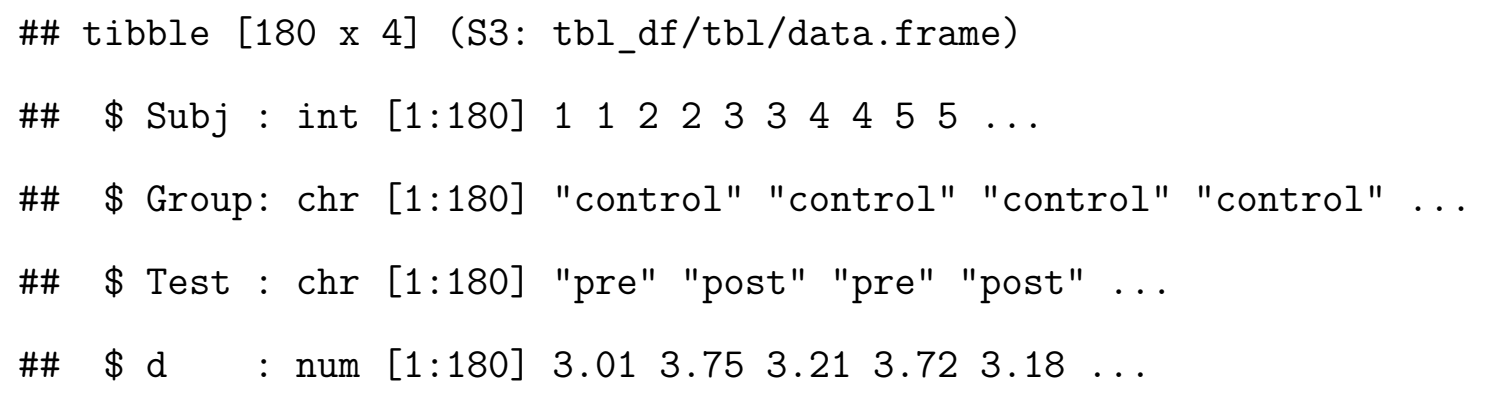




\section{Mixed ANOVA}

When ANOVA is used, we should examine the following effects:

1. For the first question, one simple effect (i.e., the comparison between pre- and post-tests in Protocol 1 group) and the simple interaction between Group (control vs. Protocol 1) and Test (pre- vs. post-tests) should be examined.

2. For the second question, one simple effect (i.e., the comparison between pre- and post-tests in Protocol 2 group) and the simple interaction between Group (control vs. Protocol 2) and Test (pre- vs. post-tests) should be examined.

3. For the third question, two simple effects (i.e., the comparisons between pre- and post-tests in the two training groups) and the simple interaction between Group (Protocol 1 vs. Protocol 2) and Test (pre- vs. post-tests) should be examined.

Noteworthy that some of the above effects are expected to be in particular directions, and, however, ANOVA obscures the directional information. Therefore, we additionally need to check descriptive statistics to make sure the directions of differences are as expected.

For the above three questions, researchers may conduct three $2 \times 2$ ANOVAs separately. Nevertheless, one caveat is that when three $2 \times 2$ ANOVAs are used, even though the variances for each condition are assumed to be homogeneous in each ANOVA, the variances of the same condition may differ in different ANOVAs. For instance, the variances of pre-test performance in the control group may be different in the two $2 \times 2$ ANOVAs for answering the first two questions. This caveat does not mean that performing three $2 \times 2$ ANOVAs is incorrect, but researchers should clarify the assumptions underlying this approach. Alternatively, we may conduct a typical 3 (Group: control, Protocol 1, vs. Protocol 2) $\times 2$ (Test: pre-test vs. post-test) mixed-ANOVA at first and then examine the above effects with specific contrasts. The 3 (Group: control, Protocol 1, 
vs. Protocol 2) $\times 2$ (Test: pre-test vs. post-test) mixed-ANOVA is conducted with library (afex) as follows:

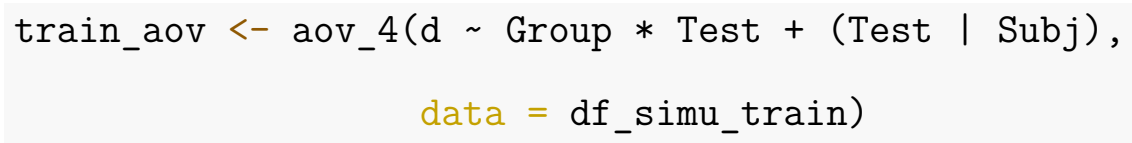

The results are not displayed as none of these effects can provide answers for the above questions. Next, we use library(emmeans) to test two simple effects of interest:

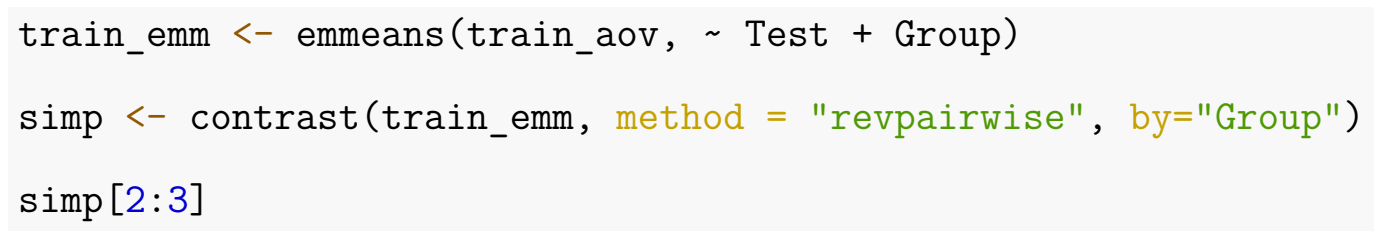

These results show that the post-test performance is better than the pre-test in both training groups. Nevertheless, we should not only use these results to draw conclusions for the three questions. Next, we conduct the simple interaction analysis:

contrast(train_emm, interaction="revpairwise")

\begin{tabular}{|c|c|c|c|c|c|c|}
\hline \#\# & Test_revpairwise & Group_revpairwise & estimate & $\mathrm{SE} d f$ & t.ratio & p.value \\
\hline \# & post - pre & protocol1 - control & 0.211 & 0.13287 & 1.593 & 0.1148 \\
\hline \# & post - pre & protocol2 - control & 0.888 & 0.13287 & 6.708 & $<.0001$ \\
\hline & ost - pre & protocol2 - protocol1 & 0.677 & 0.13287 & 5.115 & $<.0001$ \\
\hline
\end{tabular}

Simple interaction analysis results show that performance increases in Protocol 2 group are higher than the Protocol 1 and control group, respectively. No significant 
differences are observed between the Protocol 1 and control group regarding the performance increases.

With the results of both simple effects and simple interaction effects, we may claim that:

1. We fail to observe the evidence that Protocol 1 is effective. (Note that we cannot claim that Protocol 1 is not effective with the above evidence.)

2. Protocol 2 is effective.

3. Protocol 2 is more effective than Protocol 1.

\section{Custom contrasts}

Suppose another researcher wonders whether the average performance increases across the two training groups are higher than those of the control group. None of the main effects, interaction, post-hoc tests, simple effects analysis, or simple interaction analysis, can be used to answer this question. They need to apply the custom contrast of $c(1,-1,-.5$, $.5,-.5, .5)$ to the control pre-test, control post-test, Protocol 1 pre-test, Protocol 1 post-test, Protocol 2 pre-test, and Protocol 2 post-test conditions:

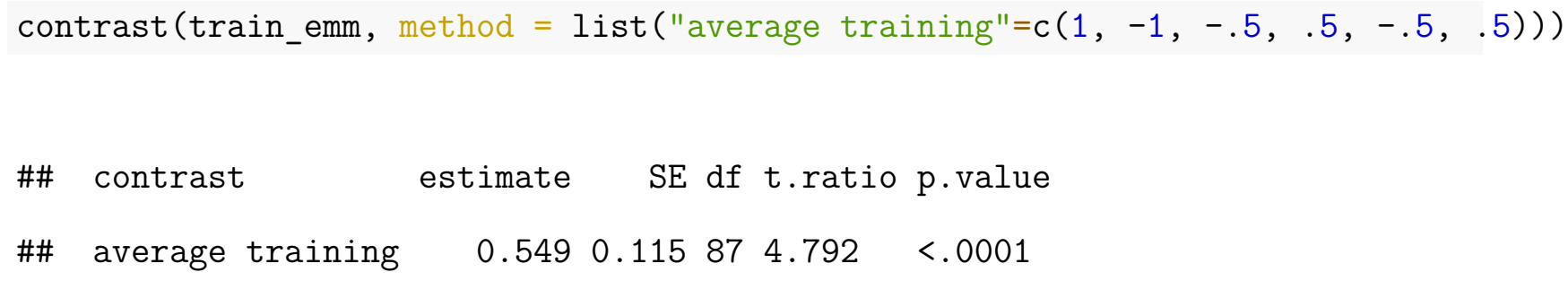

Results show that the average training performance increases are statistically higher than the control group. 
Appendix B

The complete composite face paradigm: repeated-measures ANOVA and hierarchical modeling

One of the popular paradigms measuring holistic face processing is the complete composite face task (Jin, Oxner, Corballis, \& Hayward, under review; Richler \& Gauthier, 2014). In this task, participants view two consecutive composite faces (created by combining top and bottom facial halves from different identities) and judge whether the two top halves are the same or different while ignore the bottom halves. There are mainly three independent variables in this paradigm: Congruency (congruent vs. incongruent), Alignment (aligned vs. misaligned), and Identity (same vs. different). Identity (same vs. different) refers to whether the two top (i.e., target) halves are the same or different (Figure B1). Alignment (aligned vs. misaligned) refers to whether the top and bottom facial halves are aligned or misaligned. Congruency (congruent vs. incongruent) refers to whether the situations of two top facial halves are the same as those of two bottom halves (Figure B1). Specifically, in congruent trials, when the two top facial halves are the same, the two bottom halves are also the same; when the two top facial halves are different, the bottom halves are different as well. Since in congruent trials, the situations are the same for both the top and bottom facial halves, the bottom halves are expected to facilitate the processing of top halves if participants could not ignore the bottom halves. By contrast, in incongruent trials, when the two top facial halves are different, the bottom halves are the same; when the two top halves are the same, the bottom halves are different. Thus, the bottom halves are expected to interfere with the processing of top halves if participants could not ignore the bottom halves. In other words, participants are expected to perform better in the congruent relative to incongruent trials (for aligned faces), which is also known as the Congruency effect (e.g., Richler, Cheung, \& Gauthier, 2011). Critically, this Congruency effect should be smaller for misaligned composite faces if the influence of bottom halves on the top halves mainly occurs for aligned (intact) faces, which is also known as the composite face effect (CFE; e.g., Richler, Cheung, \& Gauthier, 2011). One of the expected result patterns 
is displayed in Figure B2. For this result pattern, we should observe a significant interaction between Congruency and Alignment if we perform a 2 (Congruency: congruent vs. incongruent $) \times 2$ (Alignment: aligned vs. misaligned) repeated-measures ANOVA. This interaction is typically employed as the index of CFE (e.g., Jin, Oxner, Corballis, \& Hayward, under review; Richler, Cheung, \& Gauthier, 2011; Ross, Richler, \& Gauthier, 2015). However, a significant interaction between Congruency and Alignment does not guarantee the expected result pattern of CFE. As discussed in the main text, we also need to examine the Congruency effect in the aligned condition. Overall, we need to examine (1) whether the Congruency effect is larger than 0 in the aligned condition and (2) whether the Congruency effect in the aligned condition is larger than that in the misaligned condition.

Next, a subset of one previous study data (Jin, Oxner, Corballis, \& Hayward, under review) is used to examine whether there is evidence for CFE with repeated-measures ANOVA and hierarchical models.

\section{Repeated-measures ANOVA}

When using repeated-measures ANOVA, we need to test (1) one simple effect, i.e., the comparison between congruent and incongruent trials in the aligned condition, and (2) the interaction between Congruency and Alignment.

The 2 (Congruency: congruent vs. incongruent) $\times 2$ (Alignment: aligned vs. misaligned) repeated-measures ANOVA is conducted as follows:

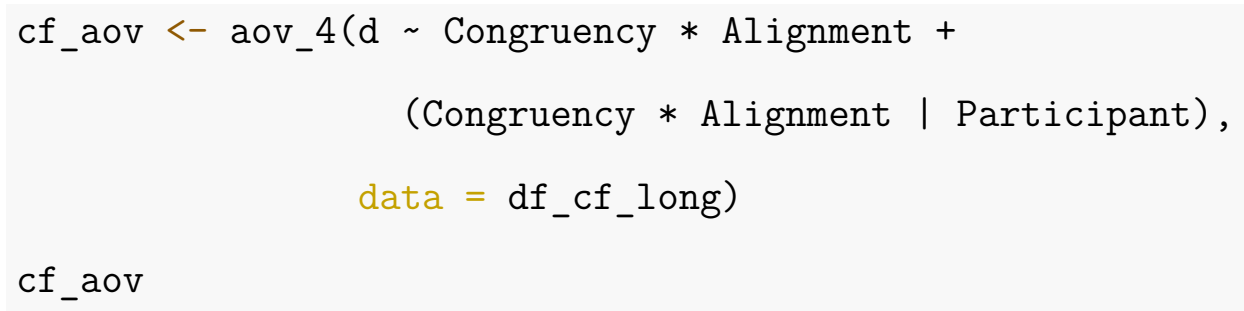

\#\# Anova Table (Type 3 tests) 


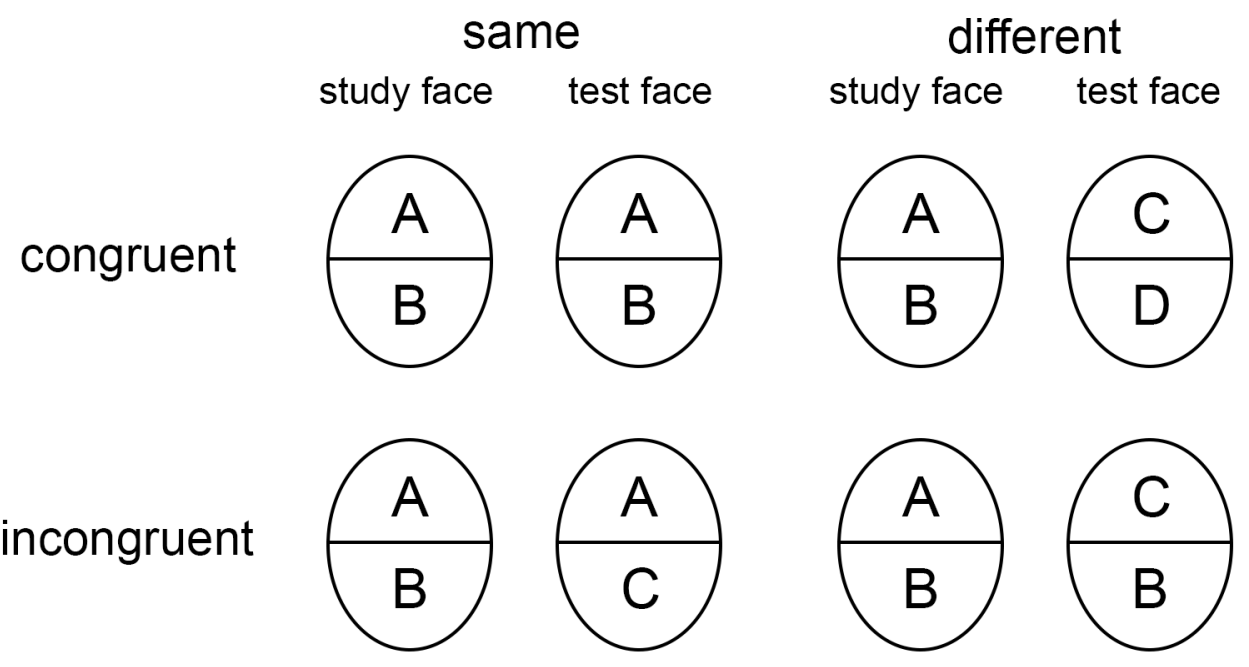

Figure B1. Experimental designs of the complete composite face paradigm for aligned faces only. On each trial, participants are instructed to judge whether the top halves of the study and test faces are the same or not. The letters denote facial identities. Same and different refers to whether the two top facial halves are the same or different. Congruency (congruent vs. incongruent) refers to whether the situations of top facial halves are the same as those of bottom halves. In congruent trials, when the two top halves are the same, the bottom halves are also the same; when the two top halves are different, the bottom halves are also different. In incongruent trials, when the top halves are the same, the bottom halves are different; when the top facial halves are different, the bottom halves are the same.

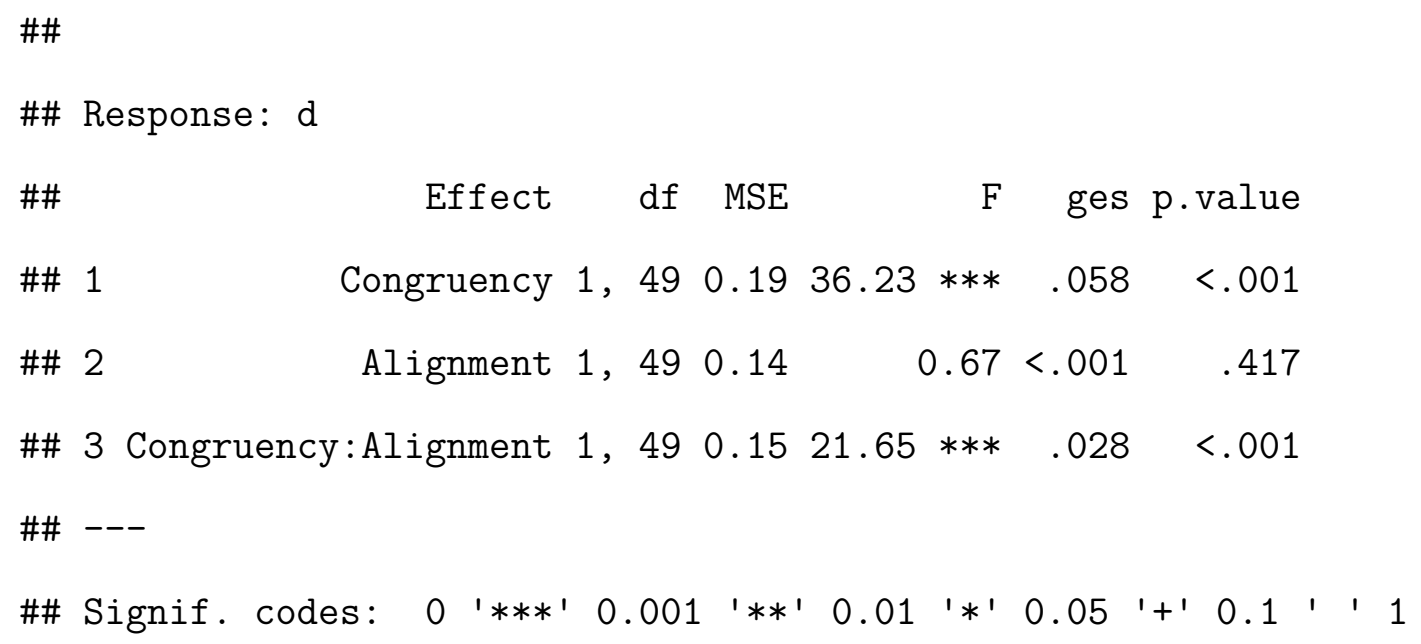




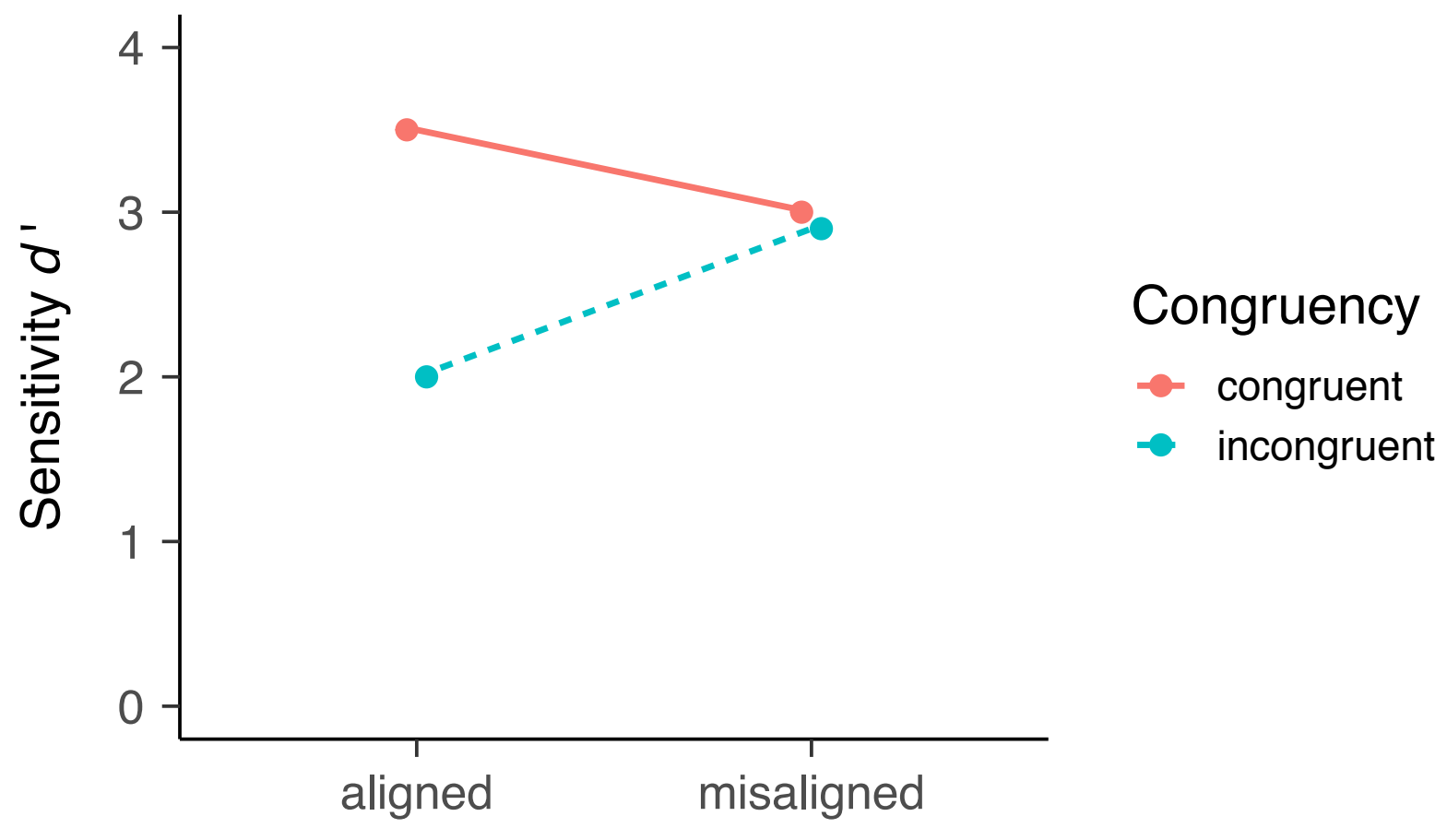

Alignment

Figure B2. One expected result pattern for the complete composite face paradigm, i.e., the composite effect. The solid (red) and dashed (green) lines denote the congruent and incongruent conditions, respectively. The same and different trials are treated as "signal" and "noise" in the signal detection theory, and both of them are used to calculate sensitivity $d$ ' to describe the behavioral performance.

We may check the interaction results in the above ANOVA table. However, we additionally have to examine the directional information from the results of the descriptive statistics. Alternatively, we may obtain the directional information by:

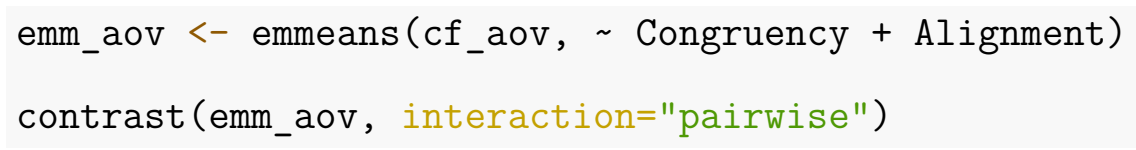


These results show that the Congruency effect in the aligned condition is larger than that in the misaligned condition. Next, we examine the simple effects according to our research question:

contrast (emm_aov, "pairwise", by="Alignment") [1]

$\begin{array}{llrrr}\text { \#\# } & \text { contrast } & \text { Alignment estimate } & \text { SE } & \text { df t.ratio p.value } \\ \# \# & \text { congruent - incongruent aligned } & 0.627 & 0.082696 .47 .593 & <.0001\end{array}$

Results of this simple effect show that the Congruency effect is larger than 0 in the aligned condition. Figure B3 displays the estimated marginal means for each condition. With both the statistically significant interaction and simple effect with particular difference directions, we may claim that the composite effect is observed in this study.

\section{Hierarchical modeling}

When hierarchical models are used, we need to examine (1) whether there is a Congruency effect in the aligned condition (i.e., whether the performance on congruent trials is better than that on incongruent trials in the aligned condition), and (2) whether the Congruency effect in the aligned condition is larger than that in the misaligned condition. For simplicity, the condition means of each participant and the intercept-only model are used in this analysis. It is typically more beneficial to conduct hierarchical models with the full random-effects structure on the trial-level data. Anyhow, this simplification would not invalidate the following demonstration. The hierarchical model is built as follows:

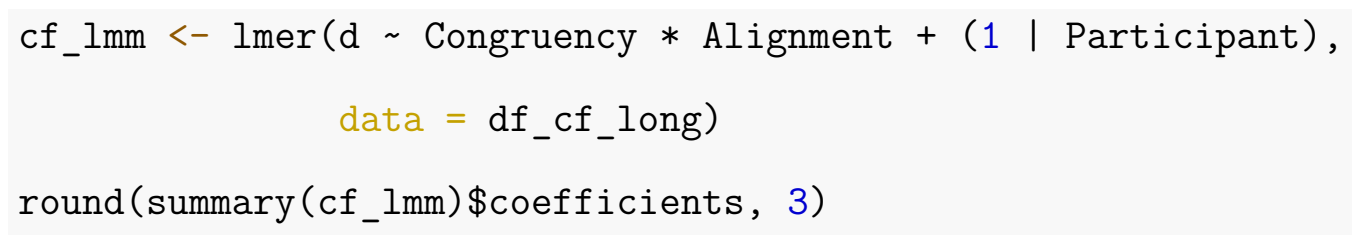




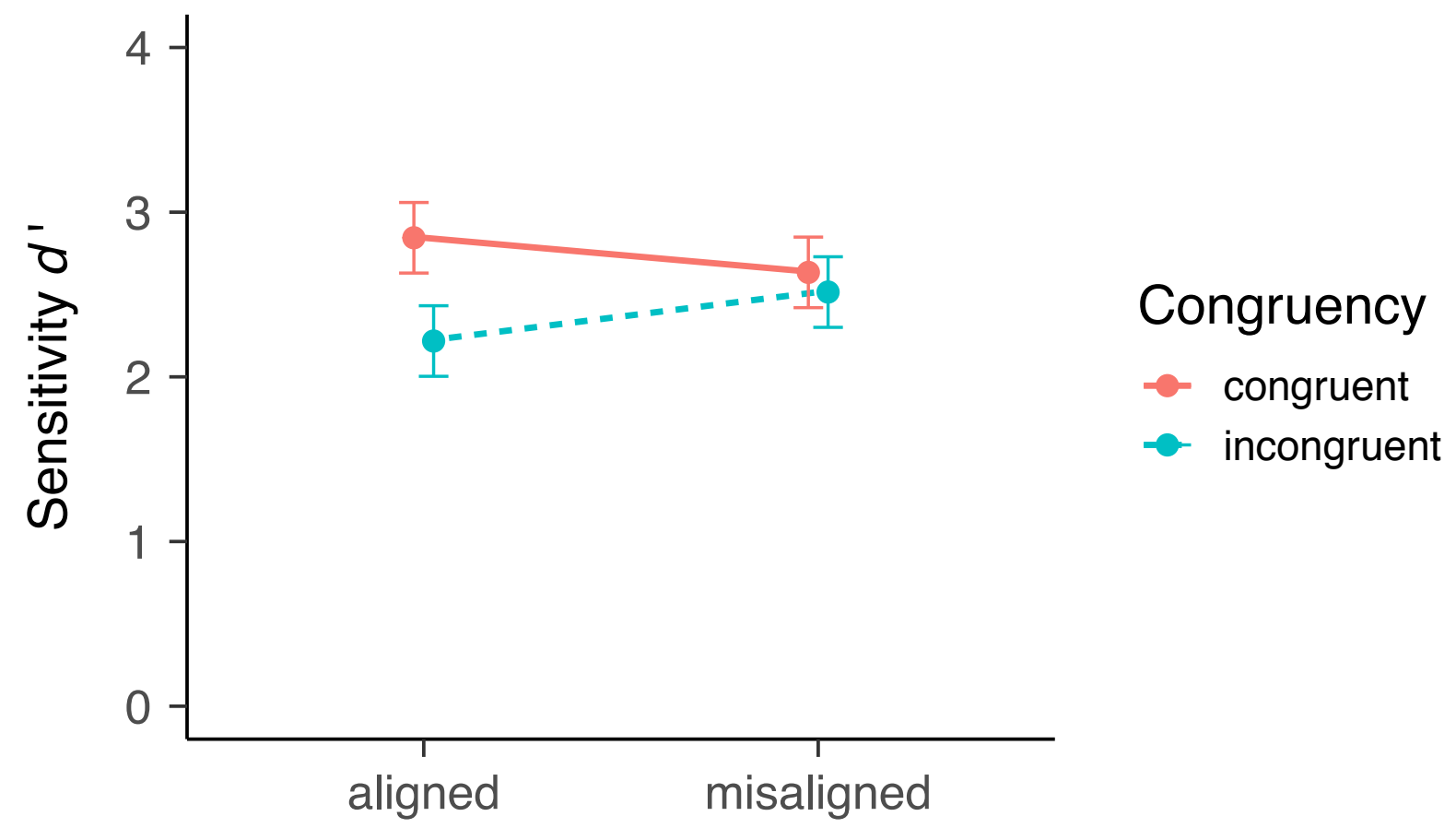

Alignment

Figure B3. Estimated marginal means of sensitivity d' as a function of Congruency and Alignment in Jin, Oxner, Corballis, \& Hayward (under review). The solid (red) and dashed (green) lines denote the congruent and incongruent conditions, respectively. Results showed that (1) higher sensitivity $d$ ' for the congruent relative to incongruent trials for aligned faces and (2) stronger Congruency effects for aligned relative to misaligned faces. The composite face effect was observed in this study. Error bars denote the $95 \%$ confidence intervals.

$\begin{array}{lrrrrr}\text { \#\# } & \text { Estimate Std. Error } & \text { df } t \text { value } \operatorname{Pr}(>|t|) \\ \text { \#\# (Intercept) } & 2.845 & 0.108 & 76.433 & 26.432 & 0.00 \\ \text { \#\# Congruencyinc } & -0.627 & 0.080 & 147.000 & -7.816 & 0.00 \\ \text { \#\# Alignmentmis } & -0.210 & 0.080 & 147.000 & -2.620 & 0.01 \\ \text { \#\# Congruencyinc:Alignmentmis } & 0.508 & 0.113 & 147.000 & 4.474 & 0.00\end{array}$

With the default dummy coding in R, Congruencyinc examines how much the performance on aligned incongruent trials is higher than that on aligned congruent trials, 
which is significantly smaller than 0. Congruencyinc:Alignmentmis examines how much the Congruency effect in the aligned condition is larger than that in the misaligned condition, which is significantly larger than 0 . In other words, the Congruency effect in the aligned condition is larger than 0 , and it is also larger than that in the misaligned condition. With this evidence, we may claim to observe the composite effect in this study. Note that since our predictions are directional, we may use one-sided instead of two-sided tests. Specifically, we may use a t-value of 1.65 instead of 1.96 as the threshold to claim statistical significance. 
Appendix C

Hypothesis-based Type I Error Rate for the composite face effect

Simulation is employed to calculate the Type I error rates at both the single test and hypothesis-based levels for examining the composite effect in the complete composite face task (Jin, Oxner, Corballis, \& Hayward, under review; Richler \& Gauthier, 2014). In each iteration, null effects are used to simulate data for a 2 (Congruency: congruent vs. incongruent $) \times 2$ (Alignment: aligned vs. misaligned $)$ within-subjects design where the population means for all four individual conditions are the same. Then the p-value of the comparison between aligned congruent and aligned incongruent conditions and the p-value for the interaction are calculated. This process is repeated 5000 times for each of ten sample sizes (varied from 10 to 1000). After simulating the data, different $\alpha$ levels for single tests are applied to each iteration. The Single Test Type I Error Rate (STER) for the simple effect and the interaction, as well as the Hypothesis-based Type I Error Rate (HER) where both the simple effect and interaction are significant, i.e., the Conjunction Type I Error Rate, are calculated. Results (Figure C1) show that STER for the interaction and simple effect match the corresponding $\alpha$, while HER is about half of the $\alpha$. For example, when the conventional $\alpha$ of 0.05 is applied to single tests, STER for both tests is about 0.05 while HER is below 0.025 . When the $\alpha$ of 0.1 is applied to single tests, STER for both tests is round 0.1 while HER is below 0.05 . Therefore, for examining the composite face effect, we may apply the $\alpha$ of 0.1 to single tests to control the HER below the conventional level of 0.05 . 


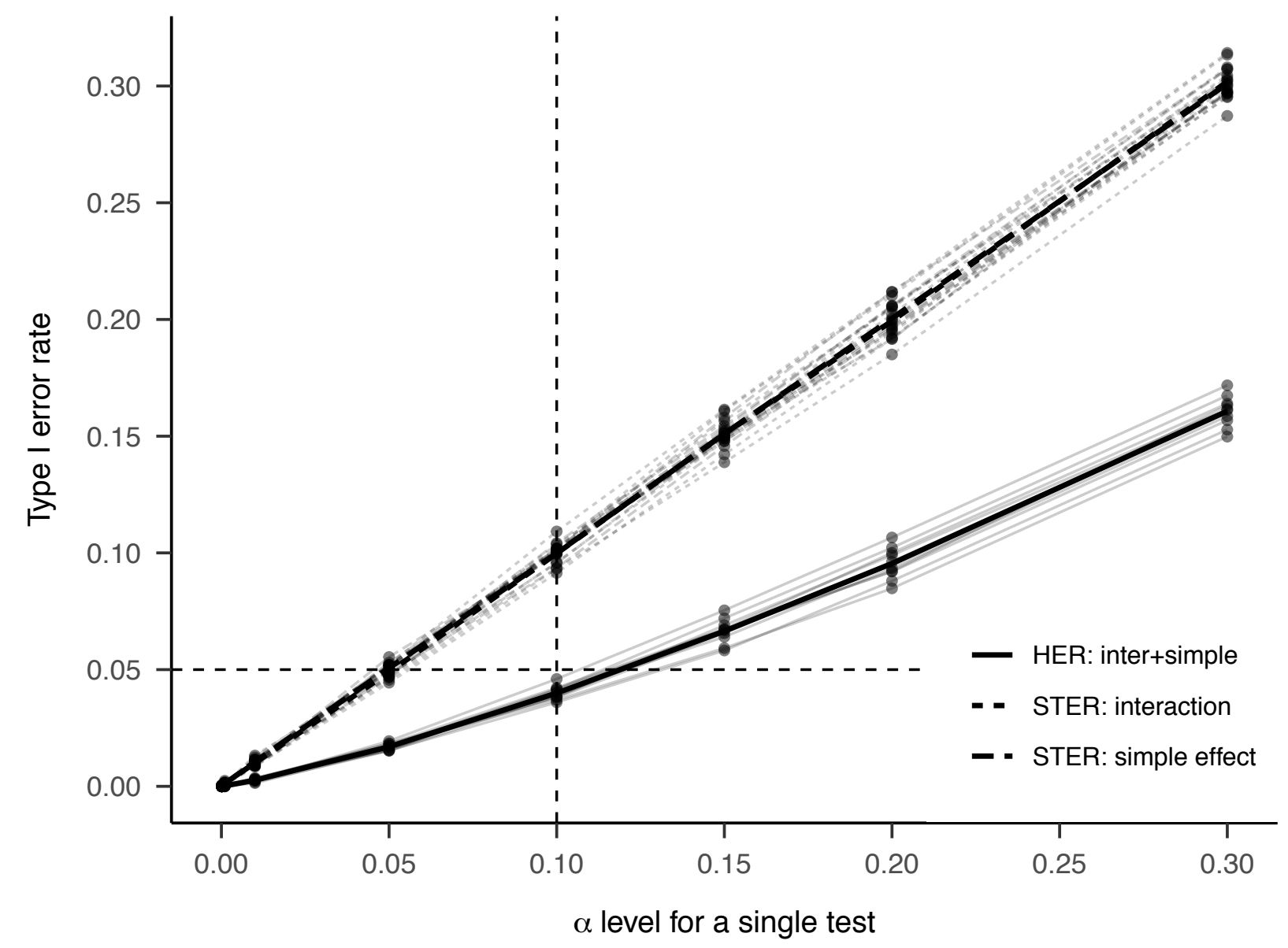

Figure C1. Type I error rates at both single test and hypothesis-based levels when different $\alpha$ is applied to single tests. The dashed lines denote the Single Test Type I Error Rate (STER) for the interaction and simple effect, respectively (the two types of dashed lines overlap with each other). The solid line denotes the Hypothesis-based Type I Error Rate (HER) for claiming the composite face effect, i.e., the Conjunction Type I Error Rate where the interaction and simple effect are significant at the same time. The gray dashed and solid lines denote STER and HER for simulated data with different sample sizes (varying from 10 to 1000), and black lines are the average error rates across sample sizes. STER and HER are similar for data with different sample sizes. STER for the interaction and simple effect match the corresponding $\alpha$, while HER is about half of the $\alpha$. For instance, when the $\alpha$ of 0.1 is applied to single tests indicated by the vertical dashed line, HER is just below 0.05, the conventional threshold indicated by the horizontal dashed line. 
Jin, H., Oxner, M., Corballis, P., \& Hayward, W. G. (under review). Holistic face processing is influenced by non-conscious visual information.

Richler, J. J., Cheung, O. S., \& Gauthier, I. (2011). Holistic processing predicts face recognition. Psychological Science, 22(4), 464-471.

https://doi.org/10.1177/0956797611401753

Richler, J. J., \& Gauthier, I. (2014). A meta-analysis and review of holistic face processing. Psychological Bulletin, 140(5), 1281-1302. https://doi.org/10.1037/a0037004

Ross, D. A., Richler, J. J., \& Gauthier, I. (2015). Reliability of composite-task measurements of holistic face processing. Behavior Research Methods, 47(3), 736-743. https://doi.org/10.3758/s13428-014-0497-4 